\title{
APOSTILLAS A LA TABLA ASTROLÓGICA BILINGÜE PUBLICADA POR P. KUNITZSCH
}

\author{
Por \\ FEDERICO CORRIENTE
}

Hace ya algunos años me comunicaba el doctor Charles Burnett, de Saint John's College (Cambridge), su trabajo sobre una singular tabla astrológica de un manuscrito de la Biblioteca Nacional de París, con su lectura tentativa de las frases árabes y sus traducciones latinas que contenía. Utilizando sus propuestas y la fotocopia del documento que amablemente me había comunicado, le hice determinados comentarios con vistas a su publicación, que él habría efectuado de no haber sabido al poco que P. Kunitzsch tenía adelantado el estudio del mismo documento, del que además había descubierto un ejemplar paralelo en la Niedersächsische Landesbibliothek de Hannover.

En efecto, no mucho más tarde aparecía la edición y estudio de dicho texto (1) por este último investigador, titulados «Eine bilingue arabisch-lateinisch Lostafel», en la Révue d'Histoire des Textes 6 (1976), 267-304, donde ponía de relieve el curioso carácter del documento, cuya exacta finalidad no es posible precisar, y su interés como bilingüe árabe (andalusí) - (bajo) latín, que el editor fecha entre 1000 y 1200 , lo que supondría el más temprano texto andalusí en grafía latina y de cierta extensión que hasta la fecha tengamos, puesto que contiene casi cien frases de dos, tres y excepcionalmente cuatro palabras que indican determinadas acciones encasilladas horizontalmente según planetas y verticalmente según signos del Zodíaco (sólo diez por corte del original copiado). En conjunto, se trata de material curiosísimo, sobre el que aún se pueden decir algunas cosas, que es lo que a continuación y en parte se intenta, tratando de hacerlo más aprovechable como fuente de conocimiento del árabe andalusí.

(1) Cuyo descubrimiento inicial agradecía a R. Lemay (v. art cit. n. 2). 
El sistema utilizado de transcripción latina es, sin duda, arcaico y casa bien con la datación sugerida, pues / $/$ / se transcribe consistentemente como sc, no $x(2), y / \%$ se refleja como $g(3)$, aunque, por otra parte, se dan varios casos de law/ representada por o (4). La imāla es de primer grado y curiosamente afecta a los reflejos de $/$ širă'/ y lašsi'rà/ (5), c parece indicar $/ \tilde{c} /$, por ensordecimiento en cauda (6), de modo sistemático en burc $=/$ búrj/, y la transcripción de /s/ es siempre $z$, más rara vez $t z$, lo que confirma un rasgo típico del iberorromance, cuya 's's' marcadamente apical impedía utilizar $s$ en dicha función y obligaba a buscar un procedimiento distinto para sugerir la predorsal, lo que más tarde desembocaría en el signo c/c. La geminación se inhibe generalmente (7). En fonética combinatoria llama la atención la solución del grupo consonántico $/ K K+K /$, que tiene algunas veces el resultado normal (8), vgr. darbi duf = /dárbǐ dúff/, darbi bok = /ḍárbǐ búq/, garzi karm = /gársǐ karm/, faltando otras veces la vocal conectiva, vgr. darb deuuema = /dárb dawwáma/, darb keber = /dárb kabár/, darb karamel = /dárb karamáli, rakz zabie = /raqș șabíyyal, scorb hgale uuedi = Išúrb 'ala wádi/, scorb nabid = Išúrb nabíd $/$, scem uuerd = /šámm wárd $/$, y dándose otras una solución $/ \mathrm{KaK}+\mathrm{K} /(9)$, vgr. en ekel harof poton -tamar haluua, etc. = lák[a]l xarúf putțún tám[a]r hálwal, etc., hikar albidia = / hik[a]r albídya/ y kezeb ganam = / kás[a]b ganám/ (pero, en cambio, kazbi zobeid = /kásbǐ șubbáyd/): en todos estos casos se da sonorante, sibilante o geminación inhibible (10), pero, ello, no obstante, el hecho llama la atención sobre posibles soluciones alternativas, a veces ya conocidas como licencia poética, a los grupos consonánticos, tal vez distribuidas según registros o variantes diatópicas o diacrónicas.

En cuanto al nivel morfosintáctico, no hay mucho que observar en un texto donde tenemos exclusivamente sintagmas preposicionales, de rección y calificativos: en éstos se advierte un uso regular de /an/conectivo ante el adjetivo, vgr., gine an tagib = /giná an táyyib/, hamen an mukteceb = /hamám an muktasíb/, faraz an dari = /farás an ḍári/ y zafagan galet =/șáf[a] an galíż (11).

Es en el aspecto léxico, y concretamente en el reconocimiento de voces

(2) Dos casos de $s$ parecen deberse a omisión gráfica de $c$

(3) Grafema utilizado también para transcribir $/ \tilde{\mathrm{g}} / \mathrm{y} / \mathrm{j} /$, e incluso para / $\mathrm{y} / \mathrm{l}$ en un caso

(4) $V$. Sketch 1.4 y especialmente 1.4.4 acerca de la monoptongación en andalusí, si bien puede en este documento proceder del oído o hábitos grafémicos de escribas romances.

(5) $V$. Sketch 1.1.1/6. Se da imāla de $20^{\circ}$ grado en /albíya/; acerca de la imåla incondicional, v. la introducción a la nueva edición El léxico árabe andalusi de $P$. de Alcalá. Zaragoza, 1988, p. IV.

(6) V. Sketch 2.19.4

(7) Incluso en los casos de $\mathrm{ir} /$ y $/ 1 /$; $\vee$ Sketch 3.2 .2

(8) V. Sketch 4.4 .3

(9) Como la señala en Sketch 4.4 .1 para / KK/ final y que se cumple, vgr, en albahar, dafar, bagal, zafag, etc.

(10) V. Gramática, métrica y texto del Cancionario hispanoárabe de Aban Quzmán, 1.2.3 y Poesía estrófica

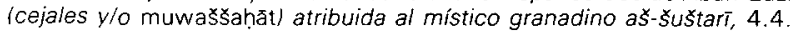

(11) V. Sketch 7.1.1. 
dejadas por Kunitzsch como irreconocibles o que pueden parecer otra cosa distinta de lo por él sugerido, donde se puede hacer una contribución más interesante a este valioso trabajo. Damos, pues, a continuación la lista íntegra de frases en su grafía latina hipotéticamente original y en transcripción fonémica dialectológica, sin más comentarios que los indispensables:

A) Signos del Zodíaco: burc alhamel athauur alieuze aceratan alezeth azunbala almizen alahagrab alcauz aliedi = /búrj alhamál -attáwr aljawzá assarațán alasád assúnbala almizán al'aqráb alqáws aljadil.

B) Planetas y astros asimilados: ascemzi = lašsámšl $/$, alkamar $=1$ alqamárl, albeder = |albádrl, alketib = |alkátib/, azohra = /azzúhral, almarech $=$ |almarrix l, almusteri = |almuštaril, almukatil = |almuqátill, alkaid $=$ lalqáyid/, ascigre $=$ |aššíral.

C) Frases:

1) Conuenientia desideria bolog emel = /bulúg (12) amál/.

2) Transportatio per loca amena nuzha fi butzten = /núzha fi bustán/.

3) Cõmestionem agni ekel harof = lák[a]l xarúf/.

4) Ascensio palme tolog fi nahla = /tulú fi náxlal.

5) Comparatione puelle scire geria = Išrá járyal.

6) Dormire in umbra rukad fi del = /ruqád fi zíll/.

7) Bibere uinum scorb hamar = /šurb xám[a]r).

8) Tangere tympanum darbi duf = /ḍárbǐ dúff/.

9) Manducare pullum anseris ekel poton = lák[a]l putțún/: en la segunda voz no procede su corrección en *būgūn "pichón». Burnett ya sugería en nota manuscrita la raíz $\{b t ̦\}$; es sin duda un diminutivo con morfema romance $\{$-ón $\}$ y armonización vocálica del andalusí /páțt/ según, vgr., Alcalá ( $v$. por raíz árabe en la nueva edición citada en n. 5 ).

10) Mille mankusos elf dirham = lálf dírham/: la segunda voz, en el mns. nuham, es de lectura problemática. Kunitzsch querría leer nuhaz $=$ Inuhās/ "(moneda de) cobre", pero mancuso era sinónimo del dinar de oro labrado (=/manqūs/): quizá pudo aplicarse al dirhem de plata (de grafía más próxima a la del mns.), pero muy improbablemente a la de cobre.

11) Fructum palmé ekel tamar = lák[a]l tám[a]r/.

12) Emere uillam scire karia = /širá qaríyya/ (13).

13) Linguam bouis panem circumdatam muhabbaza min lizen tor $=$ /muxábbaza min lisán táwr/.

14) Turnare buxium darb deuuema = /ḍárb dawwáma/.

15) Vincere inimicum dafar behgadu = /ẓáf[a]r bi 'adú/.

112) Sugerencia de Burnet en nota manuscrita de nuestra correspondencia, como en todos los casos en que se cita su opinión.

(13) V. Sketch 5.2.6. 
16) Orare (14) in absconso darta balhafi = /ḍárța balxafi/ (15).

17) Cantilenas bonas gine an tagib = /giná an țáyyib/ (16).

18) Equum bonum faraz an dari = /farás an dári/ (literalmente uentrenado", atestiguado en al-Andalús y más probable que el /naẓzāā/ que extrae Kunitzsch del Lisān, cuyo uso vulgar y en Occidente no consta).

19) Cõmestionem dulcissimam ekel haluua = lák[a]l hálwa/ (17).

20) Natare in lacu gom fi zahresc = l'áwm fi șahrijl (18).

21) Edificare domos bunien dar = /bunyán dár/.

22) Manducare gallinas ekel digega = lák[a]l dijája/ (19).

23) Comparare mulum scire bagal = /širá bág[a]l/: la lección mulum del mns. muliū y su equivalencia árabe son sugerencias de Burnett, preferibles a la lección miluum de Kunitzsch, quien por no corregir la metátesis del mns. $g$ 'abe/ recurre a un raro e improbable /'ubbāl/. Pero podría llevar razón. si fuera el garbāl del Vocabulista in arabico. (20)

24) Bibere cum amicis munedemat alihuuen = /munádamat alixwán/

25) Pellicinam madurinam faro fenek = /farú fanák/.

26) Cõmedere fructum papaueris ekel bedengen = lák[a]l badinján/.

27) Fistulare darbi bok = /dárbì búq/.

28) Flebotomare fazd $=/$ fáșd/.

29) Intrare balnium duhol hamen = /duxúl hammám/.

30) Saltationem puelle rakz zabie = /ráqș șabíyya/.

31) Cabalcare camelum rukub gemel = Irukúb jamál/ (21).

(14) Tanto Kunitzsch como Burnett son conscientes de que orare es errata por pedere, quizá a través de un petere, tal vez enmendado por malsonante si quien copiaba el latín ya no entendía el árabe, cosa indicada por otras erratas. Tampoco se puede excluir un derivado de aura como el que ha dado nuestro "orate" y el >lawțar del Vocabulista in arabico: $v$. "Notas de lexicología hispanoárabe. II. Los romancismos del Vocabulista in arabicon en Vox Romanica 39 (1980) 194-210, especialmente p. $207, n{ }^{\circ} 67$.

(15) Es curioso no se use en esta frase el más breve y castizo /fáswa/, cuyo verbo sí se refleja en cambio en un pasaje paralelo de $1021 / 15 / 4 /$ man fasá lu táht attiváb/ «(confuso como) quien ventosea bajo la ropas.

(16) $\mathrm{V}, \mathrm{n}, 12$

(17) Este es el resultado dialectal de /nalwā'/, a causa de la confusión de morfemas de femenino señalada en Sketch 5.6.2, como se documenta, vgr., en IO $102 / 12 / 4$ y $139 / 9 / 3$

(18) Voz árabe de origen persa, probablemente del pahlaví /c̄āh + rëg/ "pozo de arena".

(19) Para la palatización de la/ aquí y en el artículo de $\$ 47, v$. «Notas sobre la interferencia clásica en hispanoárabe* en Revista del Instituto Egipcio de Estudios Islámicos 21 (1981-2) 31-42, especialmente p. 33 n. 4, y "Apostillas de lexicografía hispanoárabe" en Actas de las /I Jornadas de cultura árabe e isiámica, p. 132, s.v. "Exea".

(20) A pesar de la tendencia del andalusí a vocalizar la alif inicial con /a/ frente al clásico con /i/ (v. Sketch $\mathrm{n} .841$, todos los testimonios andalusíes indican /ixwán/ sic, vgr., en Vocabulista in arabico y Alcalá): El *lahwān/ que Kunitzsch sugiere, sin apovo real paleográfico en este caso, dado lo corrupto de la grafía, se basa sólo en una mala inteligencia de la contribución de Steiger, cuyo topónimo portugués (con las grafías Alahobeines, Alahoen. Alahouen. Alohoeis, Alafoeis y Alafóes) no documenta el p. /ixwăn/, sino el dual /axawáyn/ (sic en Vocabulista in arabico, S.V. ufrater", como podria haber advertido el editor al ver con más detenimiento la interpretación allí citada de Lopes, «dois castellos fronteiros perto de Vizeun.

(21) O /jámlalli, según Alcalá. 
32) Manducare pulmentum lacte confesta ekel buliat = lák[a]l pulyáț: Es inverosímil el rarísimo/qiba/ "ventrículo", que Kunitzsch ha convertido en "callos" ante la dificultad del pasaje y a pesar de la traducción latina, no habiendo duda de que mns. bibat es errata por * buliat "poleadas, puches" (22).

33) Sonitum tubarum darb arkela: Se puede suponer que la segunda voz, nombre de instrumento musical declarado irreconocible por Kunitzsch, sea la 〉arkīla〈 del Supplément de Dozy, norteafricano/rgilal, de un nombre de unidad /nārajīla/ "coco», paralelos al persa /nargil/ o turco nargile, ambos ya con el sentido de «pipa de agua» y procedentes del sánscrito /nārikera/ «coco» (23). No podemos precisar si la metonimia que convirtió el narguilé en instrumento de viento se debió a parecido morfológico o a contagio con algún tecnicismo como el /urḡül/ egipcio, cuyo étimo parece ser el gr. órganon.

34) Plantare uites garzi karm = /gárš̌ kárm/.

35) Continentie gerilorum hikar albidie = /hik[a]r albídyal: La segunda voz es sencillamente el reflejo andalusí de /bādiya/ con imāla de $2 .^{\circ}$ grado (24).

36) Vestimenta occulata thob uuesci = /táwb wašil.

37) Vultum amabile roiat habib = /rúyat habíb/.

38) Manducare cybum de tribus rebus factum ekel mutheleth = lák[a]l mutallát/: La segunda voz es sugerencia muy plausible de Burnett para enmendar una grafía muy deturpada que Kunitzsch, contra el texto latino, convierte en uuitze $=/$ Wízza/ "ganso». Este plato es citado en lbn Quzmān 19/5/2 (25).

\section{9) Nubere tezuuig $=/$ tazwij/.}

40) Meditationem gihed $=/$ jihád/: Es evidente que la traducción latina no corresponde a este recuadro; la voz /jihád/, que Alcalá traduce "batallar por la ley», corresponde probablemente a $\$ 95$, marti[ri]um optimum, cuyo árabe a su vez parece ser de $\$ 85$, componere metrum.

41) Manducare turdum ekel zarazir = |ák[a]l zarázir $\mid$.

42) Pilleum hobuliūu kalenzuet uuesci = /qalansúwwat wašil

43) Columbam ioculatricem hamem an muktezeb: La lectura de la última palabra, irreconocible para Kunitzsch, es una sugerencia de Burnett, quien,

122) Voz documentada en Alcalá y Vocabulista in arabico (v. D. Griffin, Los mozarabismos del "Vocabulista» atribuido a R. Martí, p. 118), 10 92/6/6 e lbn Hišãm (v. Al Ahwānī, "Alfāę magribiyya min kitāb Ibn Hišām al-Laxmī fī laḥn al-'āmma" en Majalla Ma'had al-Maxtutụăt al-'Arabiyya 3 (1957) 125-57 \& 285-321, especialmente p. 23, con la mala lectura "polenta".

(23) Curiosamente, en dialecto egipcio se ha traducido correctamente esta voz, llamándose /gūza/ a una variedad primitiva del instrumento, un coco, a veces sustituido por cualquier recipiente, en cuyos agujeros se inserta la chimenea y la caña pordon de se aspira el humo a través del agua. La variedad más refinada, de vidrio, recibe el nombre persa de éste, ISišal.

124) Voz documentada en Alcalá ( $v$. \{ bdw) y, sin imāla, en Vocabulista in arabico s.v. "rusticitas», siendo de observar que funcionaba también como pl. de /badawí/ urústicon, por lo que podemos aqui traducir "enfiteusis del campo o de los labradores.

(25) $\mathrm{V}$. la explicación del pasaje en El cancionero hispanoárabe, n. 6 . 
con todo, no explicaba en sus notas su correspondencia con el latín ni el sentido que habría que dar a "palomas adquirentes". Teniendo en cuenta que ioculatores eran, en términos de Alfonso $X$, juglares o saltimbanquis, cabe pensar que se quiera aquí designar un tipo de ave con cierta habilidad para generar a su amo ganancias con arte peculiar: tal vez sea sinónimo del /hamám xaláq/ o "palomo ladrón" (26).

44) Fabricare diuersorium bunien fundec = /bunyán fúndiq/ (27).

45) Sonitum cordarum hiag tãbor = /hiyáj țanbúr/.

46) Comparare hominem imberbem scire fetien = /širá fityán/.

47) Medicinam discere: Se impone la sugerencia de Lemay de que se trata en realidad de «jugar al ajedrez», por desplazamiento de equivalencias, leyéndose legeb iscatranzi = /líb iššațránj/ (28).

48) Manducare fructum spine ekel hurscuf = lák[a]l xuršúf/ (29).

49) Venare anates zaid burac = /șáyd burák/.

50) Manducare sambucum ekel scebal = lák[a]l šáb[a]l/: No se trata de deformación morfológica del ár. /šibitt/ "eneldo", ni la voz latina quiere decir "saúco», pues es tan sólo el término bajo-latino que dio el catalán y aragonés «saboga» y gallego "samborca», sobre cuyo origen celta y parentesco con el castellano «sábalo», étimo de este /šábl/, v. Coromines, $D C E C H(30)$.

51) De domicilio contemplari loca amena nuzha min gair haria = Inúzha min gáyr xárja/, literalmente "amenidad sin salida»: Así hay que leer la deturpada grafía en su porción final, primer testimonio sobre cómo se escribiría /xárja/ en transcripción medieval, bien distinto del deforme, baciyélmico y acientífico "jarcha» que lamentablemente circula.

52) Bibere super ripam aque scorb hgale uuedi = /šúrb 'ala wádi/.

53) Carnem cum coleandro ekel tefeia = lák[a]l tafáya/(31).

54) Dare colahṽ zafag an galet =/șáf[a]" an galíz:/ "puñetazo recio", lectura tentativa de la última voz, declarada oscura por Kunitzsch.

(26) V. "Las xarajăt en árabe andalusi» en Alqanțara 8 (1987) 203-64, especialmente p. 247-8. Otra posibilidad seria leer hamem an muthabeb = /hamám an muthabbibl, basándose en Vocabulista in arabico s.v. "amarè" Jamare (con la nota "faciam quod amet me alius") y Alcalá nehib "rotoçar", si le damos a "ioculatrix" el sentido de "retozona", aunque esto se aleja más de la grafía del mns.

(27) Ajuzgar por los arabismos "alhóndiga" y ualbóndiga", las voces andalusies/fúndaq/y/búndaq/ desarroIlaron, por metanálisis del sufijo romance $\{-i k\}$, alomorfo /fúndia/ $y /$ búndiq $/$ : $V$. «Algunos sufijos derivativos romances en mozárabe" en Aula Orientalis 1 (1983) 55-60, especialmente p. 57, N. ${ }^{\circ} 4$

(28) La grafía original más probable *iscatranazi, en cuanto a la inserción de >aর en el grupo consonántico final, es tal vez una ultracorrección para disimular la asimilación de $\mathrm{ln} /$ en $/ \mathrm{j} /$, al tiempo que revela la pronunciación de ésta como $|z|$, que acabaría produciendo "ajedrez".

(29) Tal vez / xarsúf/, a juzgar por los arabismos, aunque Alcalá y el Vocabulista coinciden con la vocalización clásica.

(30) Donde se citan préstamos al árabe andalusí y norteafricano de ambas voces. Sobre la pesca del sábalo en al-Andalús hay abundantes datos; era tan apreciado que cuando lbn al-Xațib hace el parangón de Málaga y Salé, señala en contra de ésta que su sábalo sólo dura una estación (v. García Gómez, Andalucía contra Berbería. Barcelona, 1976, p. 154).

(31) En la actuai cocina marroquí, la /tfaya/ sigue llevando cilantro: $V$. Latifa Bennani-Smires, La cuisine marocaine, Casablanca s.a., p. 132; en cambio, es sustituido por perejil, sin dudar por razones prácticas, en la receta de Claudia Roden, A Book of Middle Eastern Food, Harmodsworth, Penguin 1968, p. 194, quien en cambio refiere la tradición en Marruecos de su origen morisco. 
55) Equitare elefantem rukub fil = /rukúb fíl/.

56) Manducare lac cuagulatum ekel gakid = lák[a]l 'aqíd/.

57) Fundibulare rami biuuadaf = /ramí biwaḍáf/.

58) Bibere uinum ficulneatum scorb nabid = šúrb nabíd/.

59) locari cum puella legib bigeria = /li’b bijárya/.

60) Euiscerare dentem halg adarz = |xál' aḍdárs $/$ (32).

61) Comparare diuersoirum scire fondek = /širá fúndiq/.

62) Vestire pallium libez dibeg = llibás díbaj/.

63) Hominem saltatorem gamel heuuria = l'ámil hawríyyal (33).

64) Cõmedere fungos ekel fukag = lák[a]l fuqqá'l.

65) Transire rukub albahar = /rukúb albáḥ[a]r/.

66) Trepeliatores: Kunitzsch quiere leer aquí el mns. legu'bahirag como /hu'ūb așșirā'/ «jugadores de lucha»: Aunque el pl. atestiguado de /lā'ib/ en andalusí parecer ser siempre el regular, su suposición no es imposible (34). En cambio, es menos probable su lectura de la última palabra, en primer lugar por falta de documentación de espectáculos de lucha en al-Andalús, quizá mal vistos por los alfaquíes como /taḡr/ o riesgo injustificado, pero especialmente porque es mucho más probable una lección /ixráj/ «representación, actuación o remedon (35) que casaría bien con la suposición de una alteración del latín tripodiatores.

67) Monochordum mensurare darb keber = /dárb kabár/.

68) Bibere mulsum scorb beneca = /šúrb bináča: La lectura de la problemática palabra final es tentativa, basada en el andalusí /bináča/ del Vocabulista in Arabico y Alcalá, al parecer un tipo de orujo (36).

(32) Acerca de $/$ KiKK/ $>\mid K a ́ K K /$, v. Sketch 5.1.3

133) Acerca de esta voz, v. "Nuevos berberismos del hispanoárabe» en Awrãa 4 (1981) 27-30, especialmente p. 26, cuyas etimologías sigo manteniendo tentativamente, aunque pensando ahora que la agachadilla y el giro no son los de una zambullida, sino hechos por los danzarines negros durante su baile y al hacer girar en torno a su cabeza los flecos u otro objeto, como aún puede verse en Marruecos. También se podría mantener el mns. heuueria = /hawwāriyyal, partiendo de una vez como el marroquí thewwar/ "eje".

(34) A la vista de los pls. $\{1 \mathrm{i} 22$ ú3 $\}$ de singular $\{1$ á2i3 $\}$ recogidos en Gramática métrica y texto... 2.4.2. 1.2. al parecer procedentes del árabe antiguo $\{1 \mathrm{u} 2 \mathrm{u} 3\}$, aunque lo más probable es que aquí haya que leer sencillamente $/ l i$ "b/ = * legib «juego" no «jugadores», pues las equivalencias latino-árabes de este documento no son tan estrictas.

(33) La documentación andalusí de esta voz comprende: a) El famoso pasaje de Aš-Sunqundī, ya recogido por el Supplément de Dozy bajo /aarawil, y mencionado por Garcia Gómez en Andalucía contra Berbería, p. 131, donde se cita /ixrāi al-qarawī/ como un género de diversión cultivado hábilmente por naturales de Úbeda, si bien no parece probable que se trate de un juego de manos, como ha puesto de relieve J. T. Monroe en "Prolegomena to the Study of Ibn Quzmān" en El romancero hoy: Historia, Comparatismo, p. 87, al analizar el cejel 12, donde se habla de un /qarawi/ que se ha quedado parado. dentro de una actuación juglaresca, y b) Ašs-Suštari 8/1/4, donde tenemos /ixráj alqawálib/ "producción de moldes", quizá "remedo" (a juzgar por el giro /șabb aiqawălib/, en el Supplément) que, curiosamente, aparece también aquí como en aš-Šuqundi en conexión con la voz /ințibā' $/$, por lo que sugerimos la siguiente lectura e interpretación de su cejel 8/1/4 y 5: / law ánna bințibái wixráj qawálibi / / win síbtu minka xáiwa, tansúb maxálibil: "Y a pesar de mi maestría y habilidad en remedos, si contigo me hallo a solas, se me enredan las zarpasn. De todo esto cabe suponer que la actividad llamada (ixráj/ en el recuadro que estudiamos es de tipo coreo-dramático.

(36) Otra posibilidad sería la presencia aquí del andalusi (banín/ «sabroso» (documentado en Supplément). 
69) Venare cum accipitre zaid bi catam = /șáyd biqațám/ (37).

70) Sedere in nuptiis scuhud gorz = /šuhúd 'úrs/.

71) Manducare perdices ekel hagal = lák[a]l hajál/.

72) Audire liram zamag rebeb = /samá rabáb/.

73) Preposituram accipere: Falta el equivalente árabe, que podría ser el mismo que el de $\$ 90$, ordinem episcopalem, cuyo ininteligible ekelzir podría ser tekdim, voz que usa Alcalá (=/taqdím/) tanto para "obispado" como para "maestrazgo".

74) Pingere palacium tezuuit megliz = /tazwíq majlís/.

75) Fabricare turrim bunien cazaba = /bunyán qașábal.

76) Ambulare per eclesias: Kunitzsch prefiere leer ecelas, lo que dificulta la inteligencia de la porción final. Semánticamente, no está claro «andar por habitacioncitas"; en cambio, con la otra variante podemos suponer que la voz final ha sido amputada como en otras casillas (vgr. 44,61 y 74) y sugerir una lectura * mezir ile alhek[al] = /masír ila alháykal/ «ir al templo».

77) Venari leporem zaid aranib = /šáyd aránib/.

78) Soluere carceratum itlac mahabuz = /ițláq maḥbús/.

79) Desiderare gra[tiam] dei: Sin equivalente árabe.

80) Sanare infirmum ifaca min algilla (38) = lifáqa min al'illa/ (39).

81) Comparare unguentum scire miar = /širá miyár/: La segunda palabra, que no reconoce Kunitzsch, quiere decir "provisiones" y tal vez ha sido confundida por el traductor con /mayrūn/ «santo óleo», que podía ser muy familiar a un clérigo mozárabe.

82) Ad longinquam terram ire duhol mizar $=/$ duxúl miș [a]r/.

83) Manducare cucumeres: Falta el equivalente árabe, desplazado a §94 eheleriha, que se leerá ekel hiar = lák[a]l xiyár/.

84) Legere libros: Falta equivalente árabe.

85) Componere metrum: Su equivalente árabe está desplazado a $\S 96$ gamelnadra, que se leerá ${ }^{*}$ gamel nadm = I'amál náẓm/.

86) Disputare cum sapiente: Falta el árabe; quizá era * gamel munadara = /'amál munáżaral, que habría sufrido haplografía por su parecido y cercanía al $\$ 96$.

87) Comparare subtulares scire ahfef = /širá axfáf $/$.

88) Apprehendere musicam: Es erróneo su equivalente árabe, que repite el $\S 52$.

pero la grafía original parece indicar una voz más larga y por otra parte, faltaría el /an/ conectivo excepcionalmente en este texto.

(37) Forma conservadora, más antigua que la atestiguada por el arabismo "alcotán». V Sketch 1.1.9 y 2.4.1.

(38) Mns. abilla, pero no es el único caso de $\underline{b} \geqslant \mathrm{~g}$ en estos materiales.

(39) No es sorprendente que la forma IV haya adquirido sentido transitivo en este verbo, pues el dialecto la eliminó como en la mayoría de los casos, creando el \}nufüq $\{\sim\}$ fiqt $\{$ del Vocabulista s.v. "surgere" (con nota «de ebrietate vel infirmitate»). Al introducir los círculos cultos o semicultos la IV, también citada a continuación en dicha obra, era natural se tomase por causativa. 
89) Odorem florum scem alzuzen = /Šámm assussán/.

90) Ordinem episcopalem: $\vee . \S 73$, debiendo excluirse la lectura de Kunitzsch * lakl șir/ "comer pescaditos salados", que extrae una rarísima voz del diccionario clásico y supone en episcopalem un resto de pisces.

91) Odorem rose scem aluuard = Isámm alwárd $/$.

92) Aperire castellum iftitah hezan = /iftitáh híș[a]n/. Obsérvese el arabismo semántico aperire $=$ conquistar .

93) Tangere organum darb karamel = /dárb karamál/.

94) Desiderium uite eterne: Su equivalente árabe corresponde a $\S 83$, como se ha indicado, no convenciendo la lectura de Kunitzsch /akl arha/ «comer terneran, pues es muy rebuscado suponer que uite sea resto de vitella o vitula.

95) Comparare boues kezeb ganam = /kás[a]b ganám/.

96) Martiū/Maritū optimum: Debe leerse martyrium, correspondiente a $\$ 40$, mientras su árabe es de $\S 85$.

97) Parare uiscum kazb zobeid = /kásb șubbáyd/ (40).

98) Ascendere turrim rukub tzor = /rukúb súr/.

99) Vestire camisiam lebez kaba = /libás qabá/ (41).

100) Invenire repositum Vgud kenz = /wujúd kánz/.

De resultas de esta revisión, deben suprimirse del repertorio léxico de las pp. 297-9 las voces arha, iwazza, büğūn, šibitt, išfā', șir, 'ubbäl, nuhās, nazzărr. nūr y giba añadir /arkíla/, /ila/ (?), /bídya/, /bulúg/, /bágl/, /pulyáț/, /bináča/ (?), /puttún/, /mutallát/, /xárja/, /ixráj/, /xiyár/, /dírham/ (?), /Sábi/, /Šuhúd/, /dáril, /țáyyib/, I’íla/, Igalíż, gáyr/, /fityán/, /taqdím/ (?), /muktasíb/, /miyár/, /názm/ y /haykal/ (?), hacier.do asimismo los ajustes necesarios a la fonética dialectal en otros casos y separándose las entradas compuestas, lo que añade a la lista /lisán/, /táwr/, /habíb/ y /țanbúr/, arrojando un total de 141 voces seguras, salvo las marcadas con interrogación.

El documento publicado por P. Kunitzsch es, pues, una importante contribución al conocimiento de las fases más antiguas del andalusí, especialmente en el aspecto léxico, y deberá ser tenido en cuenta en adelante en los estudios de este haz dialectal, una vez aquilatada la exactitud de sus materiales.

(40) No sólo figura esta voz en el Vocabulista, sino también en 10 49/6/2.

141) Aquí se rechazará un posible /káppa/ "capa», no como mozarabismo, sino por divergencia semántica e incluso fonética, pues $/ \mathrm{p} /$ es mantenida en / puțț́n/ (aunque no en /pulyáțt). 